\title{
A Precision Capacitance Cell for Measurement of Thin Film Out-of-Plane Expansion-Part III: Conducting and Semiconducting Materials
}

\author{
Chad R. Snyder, Member, IEEE, and Frederick I. Mopsik
}

\begin{abstract}
This paper describes the construction, calibration, and use of a precision capacitance-based metrology for the measurement of the thermal and hygrothermal (swelling) expansion of thin films. It is demonstrated that with this version of our capacitance cell, materials ranging in electrical properties from insulators to conductors can be measured. The results of our measurements on p-type $\langle 100\rangle$-oriented single crystal silicon are compared to the recommended standard reference values from the literature and are shown to be in excellent agreement.
\end{abstract}

Index Terms-Capacitance cell, coefficient of thermal expansion (CTE), guarded electrode, high sensitivity displacement, inner layer dielectrics, polymers, thin films.

\section{INTRODUCTION}

$\mathbf{T}$ HE coefficient of thermal expansion (CTE) is a key design parameter in many applications. It is used for estimating dimensional tolerances and thermal stress mismatches. The latter is of great importance to the electronics industry, where thermal stresses can lead to device failure. For accurate modeling of these systems, reliable values are needed for the CTE.

Traditionally, displacement gauge techniques such as thermomechanical analysis (TMA) have been utilized for determining the CTE. However, standard test methods based on these techniques are limited to dimensions greater than $100 \mu \mathrm{m}$ [1], [2]. This is problematic for materials which can be formed only as thin layers (such as coatings and certain inner layer dielectrics). Additionally, there is some question as to whether values obtained on larger samples (bulk material) are the same as those obtained for thin films, even when the effects of lateral constraints are included in the calculations [3]-[5].

It has long been recognized that capacitance-based measurements, in principle, can offer the necessary resolution for these films [6]-[8]. For a pair of plane-parallel plate capacitors, if the sample is used to set the spacing of the plates $d$ while being outside of the measurement path, then for a constant effective area of the plates $A$, the capacitance in a vacuum $C_{\text {vac }}$ is given by the well-known equation

$$
C_{\mathrm{vac}}=\frac{\epsilon_{\mathrm{0}} A}{d}
$$

Manuscript received March 20, 2000; revised May 29, 2001. This work was supported by the NIST Office of Microelectronics Programs.

The authors are with the Polymers Division, National Institute of Standards and Technology, Gaithersburg, MD 20899-8541 USA.

Publisher Item Identifier S 0018-9456(01)08740-X where $\epsilon_{0}$ is the permittivity of free space $\left(\epsilon_{0}=8.854 \mathrm{pF} / \mathrm{m}\right)$. With the sample outside of the measurement path and only air between the electrodes, the vacuum capacitance is obtained from the measured capacitance $C$ by

$$
C_{\mathrm{vac}}=\frac{C}{\epsilon_{\mathrm{air}}}
$$

where $\epsilon_{\mathrm{air}}$ is the dielectric constant of air.

In three previous papers, the design and data reduction techniques were presented for our three-terminal capacitance-based metrology for thin polymer film measurements. The first paper (I) described the initial design based on gold-coated Zerodur [9]. ${ }^{1} 2$ However, several problems were encountered. It was discovered that Zerodur displays ferroelectric behavior, with an apparent Curie temperature of $206^{\circ} \mathrm{C}$ as determined by fitting with a Curie-Weiss law. The rapid change in the dielectric constant of the Zerodur along with a coupling from the central contact through the guard gap to the high electrode created an apparent negative thermal expansion [10]. The second problem with the initial design was with the gold coating. This coating had the tendency to "snow plow" when scratches formed in the surface creating raised areas which would result in shorts when measurements were performed on thin samples. The second problem with the gold was that it underwent mechanical creep under loading.

To resolve these problems, a new electrode was designed from fused quartz coated with nichrome. A groove filled with conductive silver paint was added to the back side of the bottom electrode around the central contact to intercept any field lines between the central wire contact through the guard gap to the high electrode. The new design was described in the second paper (II) along with thermal expansion measurements on $\langle 0001\rangle$-oriented single crystal sapphire $\left(\mathrm{Al}_{2} \mathrm{O}_{3}\right)$ and a $14-\mu \mathrm{m}$ thick inner layer dielectric material [10]. It was recognized in II that the data reduction was simple as long as the air filling the gap between the capacitor plates was dry. However, to expand the utility of the capacitance cell to hygrothermal expansion (i.e., swelling in a humid environment), the third paper (III) described the data reduction techniques necessary for use of the capacitance cell under humid conditions [11].

\footnotetext{
${ }^{1}$ Schott material having a CTE of less than $0.05 \times 10^{-6} \mathrm{~K}-1$

${ }^{2}$ Certain commercial materials and equipment are identified in this paper in order to specify adequately the experimental procedure. In no case does such identification imply recommendation or endorsement by the National Institute of Standards and Technology, nor does it imply that the items identified are
} necessarily the best available for the purpose. 


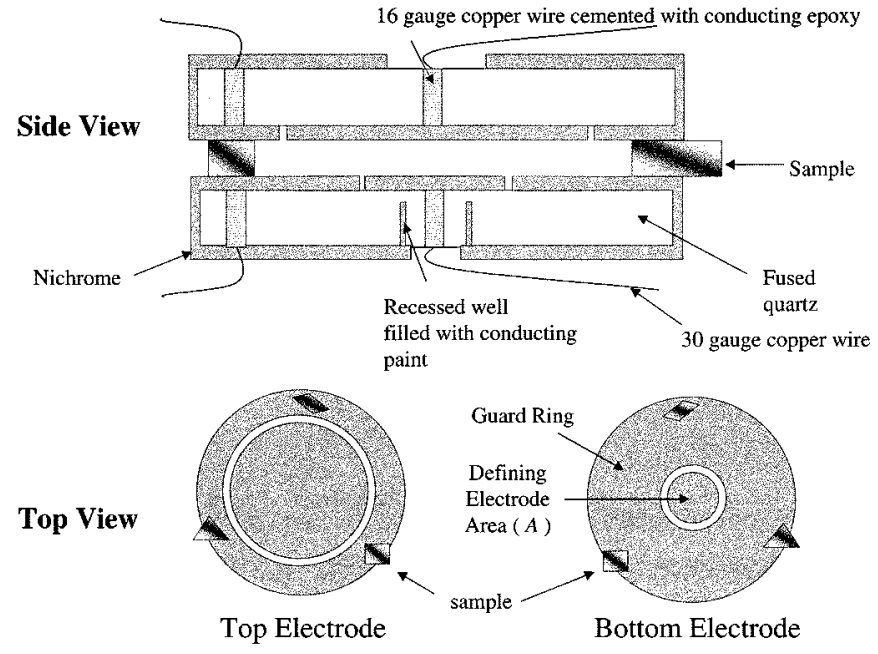

Fig. 1. Schematic of the electrodes. Note that the shaded areas correspond to the nichrome coating.

The resolution of the instrument was determined in II and III. For dry, isothermal conditions, the capacitance cell can measure relative changes in thickness on the order of $10^{-7}$, for a $0.5-\mathrm{mm}$ thick sample; this corresponds to a resolution on the order of $5 \times 10^{-11} \mathrm{~m}$. Under dry conditions in which the temperature is changed, the reproducibility of a relative thickness change (e.g., for CTE measurement) is on the order of $10^{-6}$. Finally, under humid conditions, the ultimate resolution is primarily a function of temperature - the actual values of which are given in III.

In II, a deficiency was recognized in the design. Neither semiconducting or conducting materials could be used as the material for testing. This was especially the case for silicon, which forms a Schottky barrier with nichrome and acts as a voltage rectifier. Additionally, because of the nature of the interface, the $1 \mathrm{kHz}$ measurement frequency generates ultrasound which results in the epoxy contacts being shaken loose. We mentioned briefly in II that if the top electrode had a guard ring added, the sample could be held at zero potential and this would no longer be a problem. To demonstrate this, we constructed such a capacitance cell - the design and testing of which are described in this paper.

\section{Capacitance Cell Design}

\section{A. Electrode Design}

Because the construction of the electrodes was thoroughly described in II, a less detailed description will be given with emphasis on the changes in the design. The electrodes were constructed, as before, in the following manner (see Fig. 1). $10 \mathrm{~cm} \times 2 \mathrm{~cm}$ cylindrical blanks of fused quartz were ground and polished to optical flatness. Small holes were drilled through the center of each blank so that 16 gauge wire could be inserted into them. The wires were then cemented with a conducting epoxy (resistivity of $4 \times 10^{-4} \Omega \mathrm{cm}$ at $25^{\circ} \mathrm{C}$ ). A second hole and wire were then added to each blank approximately $0.75 \mathrm{~cm}$ from the edge of the blanks. A coating of nichrome was then added such that it covered all surfaces except for a small area around the back of the blanks. A guard gap was scribed on both the top and bottom electrodes such that no

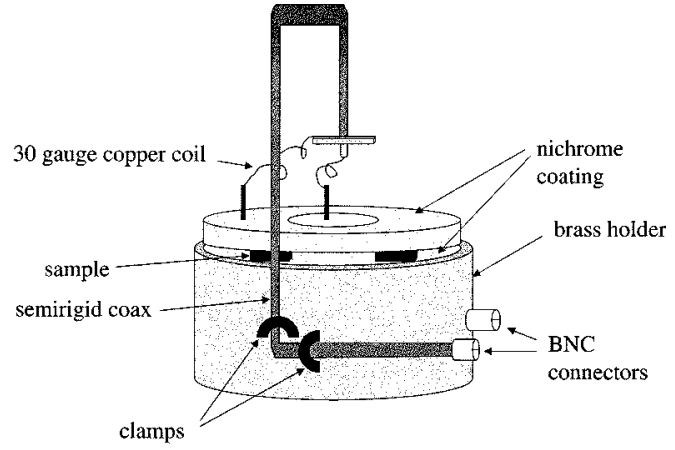

Fig. 2. Schematic of the assembled capacitance cell.

material was raised which could cause a short. On the bottom electrode, the guard gap was scribed on a $3 \mathrm{~cm}$ diameter, and on the top electrode it was scribed on a $6 \mathrm{~cm}$ diameter. In the bottom electrode, a $1 \mathrm{~cm}$ diameter well was cut into the back of the blank which extended to within $5 \mathrm{~mm}$ of the front surface. This well was then filled with a thin conductive silver paint. The paint connected the outer guard ring's metallization to the edge of the well.

\section{B. Cell Assembly and Capacitance Measurements}

The holder described in II was employed for the modified cell. In this version of the capacitance cell, both conductors of the semirigid coaxial line were connected to the top electrode. The center connector and braid were connected to the center area and outer guard ring, respectively, by fine 30 gauge wire coils. The coils were terminated with center female contacts from $50 \Omega$ BNC connectors, which could be easily connected/disconnected to the 16 gauge tinned copper wire that was epoxied into the electrodes. A schematic of the assembled cell is shown in Fig. 2. The female BNC connector on the brass holder (bottom electrode) was connected to the low terminal, and the female BNC connector on the semirigid coaxial line was connected to the high terminal. All connections from the capacitance cell to the bridge were performed using Teflon insulated low noise cables.

The capacitance measurements were obtained using a commercial automated three-terminal capacitance bridge which uses an oven-stabilized quartz capacitor and has a cited guaranteed relative resolution of better than $5 \times 10^{-7} \mathrm{pF} / \mathrm{pF}$ for the range of capacitances used with this cell (Andeen-Hagerling 2500 A $1 \mathrm{kHz}$ Ultra-Precision Capacitance Bridge with Option E). (Note that the "useful" relative resolution is suggested by the manufacturer to be typically a factor of 10 or more better that the cited relative resolution.) The capacitance bridge's calibration was verified against a National Institute of Standards and Technology (NIST) calibrated standard reference capacitor-the difference between the two was within the capacitor's uncertainty.

All measurements were performed in a temperature/humidity chamber equipped with a $-90^{\circ} \mathrm{C}$ dew point air purge. The cell was equilibrated at each temperature until the relative fluctuations in the vacuum corrected capacitance were no more than $10^{-7} \mathrm{pF} / \mathrm{pF}$. Barometric pressure was monitored using a digital pressure sensor with a manufacturer's stated uncertainty of 
$0.1 \mathrm{~mm} \mathrm{Hg}(13 \mathrm{~Pa})$. As stated previously in II, the temperature of the cell was calibrated in terms of the chamber temperature with a resistance temperature device (RTD) mounted to the cell with thermally conducting paste. The RTD was calibrated against a NIST certified ITS-90 standard reference thermometer. As in II, because we are using a dry air purge, we can use the ideal gas law correction to determine the molar volume of the air $v_{\text {air }}$ to calculate $C_{\text {vac }}$

$$
v_{\text {air }}=\frac{R T}{p}
$$

where

$T$ absolute temperature;

$p$ pressure;

$R \quad$ gas constant $\left(R=8.314507 \mathrm{~L} \mathrm{kPa} \mathrm{mol}^{-1} \mathrm{~K}^{-1}\right)$ [12]. From this and the value of the molar polarization of dry air obtained from the literature, $P=4.31601 \times 10^{-3} \mathrm{~L} / \mathrm{mol}$ [13], the dielectric constant of the air separating the electrodes is

$$
\epsilon_{\mathrm{air}}=\frac{2\left(P_{\mathrm{air}} / v_{\mathrm{air}}\right)+1}{1-\left(P_{\mathrm{air}} / v_{\mathrm{air}}\right)}
$$

\section{MEASUREMENTS}

\section{A. Cell Calibration}

To use (1) to calculate the thickness of the sample, the effective area must be known. To determine this value as a function of temperature, as in II, we calibrated the area and area expansion through the use of Zerodur spacers with thicknesses of approximately $2.0 \mathrm{~mm}$. As in II, the actual dimensions of the Zerodur spacers were measured in a ball to plane configuration with a specially designed caliper equipped with a linear voltage displacement transducer (LVDT) that had a resolution of $\pm 1 \times 10^{-4} \mathrm{~mm}$. The cell was assembled with the Zerodur spacers using the sample preparation described in II. Measurements were performed at $0{ }^{\circ} \mathrm{C}, 25^{\circ} \mathrm{C}, 50^{\circ} \mathrm{C}, 75^{\circ} \mathrm{C}, 100{ }^{\circ} \mathrm{C}$, $125^{\circ} \mathrm{C}$, and $150^{\circ} \mathrm{C}$. The cell was cycled through this range of temperature three times, and the values for $C_{\text {vac }}$ were determined for each run after averaging all the properties over approximately $1 \mathrm{~h}$ using $10 \mathrm{~s}$ increments (a total of 360 data points) after equilibrium was achieved. The area $A$ was calculated using the room temperature thickness measurements and the $25^{\circ} \mathrm{C}$ value for $C_{\text {vac. }}$. All subsequent determinations of $A$, at higher and lower temperatures, were corrected for the slight expansion and contraction of the Zerodur as a function of temperature $\left(\alpha_{Z \text { erodur }}=0.05 \times 10^{-6} \mathrm{~K}^{-1}\right)$. The results of the effective radius of the electrode as a function of temperature are plotted in Fig. 3.

\section{B. $p$-Type Doped $\langle 100\rangle$ Single Crystal Silicon}

To demonstrate the ability of the cell to measure silicon and to provide accurate values for thermal expansion, a 0.6 - $\mathrm{mm}$ thick wafer of single-side polished, back side stress relieved, p-type, $\langle 100\rangle$-oriented single crystal silicon with a resistivity of $15 \Omega$ $\mathrm{cm}$ was broken (by scribing) into three pieces. Each piece was approximately $1 \mathrm{~cm}^{2}$. The pieces were then cleaned with ultra pure distilled water and ethanol. The cell was assembled in the same fashion as was described in II and was placed in a vacuum

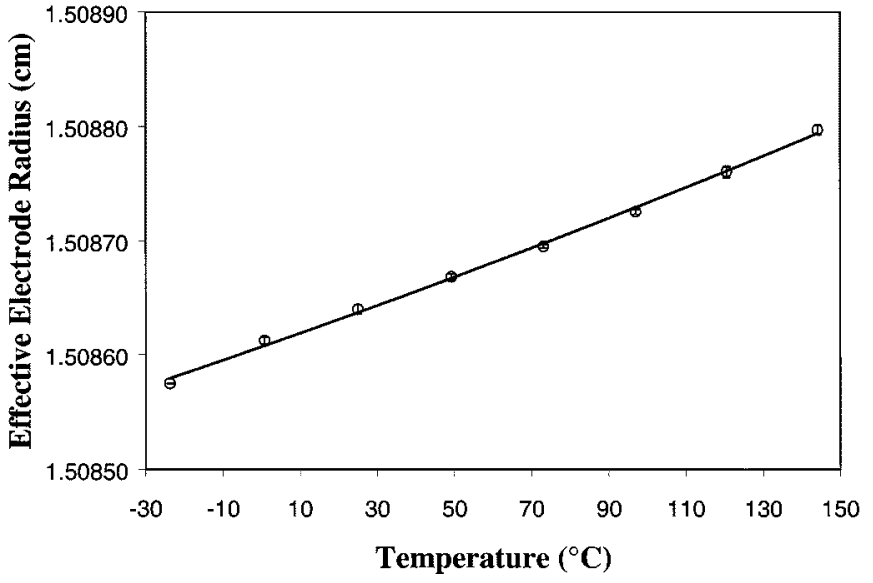

Fig. 3. Effective radius of the bottom electrode as a function of temperature obtained by measurements using Zerodur and correcting for its slight expansion. (The error bars correspond to the best estimate of two standard deviations in the experimental uncertainty-type A errors only. Previous measurements with the capacitance cell suggest that type B errors can be assumed to be zero.)

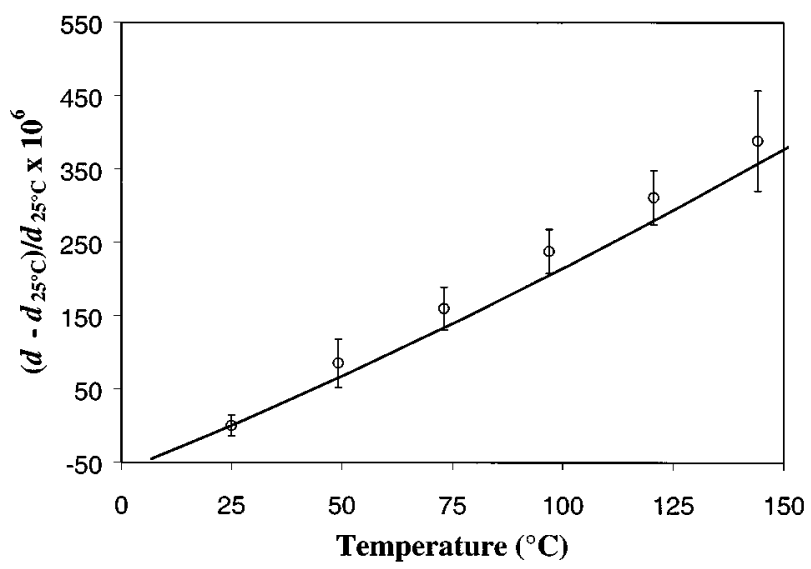

Fig. 4. Relative expansion of the $\langle 100\rangle$-oriented single crystal silicon as a function of temperature. The line is a plot of the data from [14]. (The error bars correspond to the best estimate of two standard deviations in the experimental uncertainty-type A errors only. Previous measurements with the capacitance cell suggest that type B errors can be assumed to be zero.)

oven at ambient temperatures for approximately $1 \mathrm{~h}$ to effectively wring the sample. ${ }^{3}$. Measurements were performed at 25 ${ }^{\circ} \mathrm{C}, 50{ }^{\circ} \mathrm{C}, 75^{\circ} \mathrm{C}, 100{ }^{\circ} \mathrm{C}, 125^{\circ} \mathrm{C}$, and $150{ }^{\circ} \mathrm{C}$, a minimum of two times each. (Note: No point was taken at $0{ }^{\circ} \mathrm{C}$ due to problems with the compressor in the environmental chamber.) The wafer thickness was determined using the effective radius versus temperature data shown in Fig. 3. The results of this analysis are shown in Fig. 4 along with the recommended expansion data on silicon obtained from [14]. It should be noted that the standard reference data was defined relative to $20^{\circ} \mathrm{C}$ whereas we have measured, for convenience, relative to $25^{\circ} \mathrm{C}$. Therefore, the standard reference, relative expansion data was shifted in Fig. 4 by an amount $S$ equal to

$$
S=\left(\alpha_{T}\right)(-5 \mathrm{~K})
$$

where $\alpha_{T}$ is the CTE at temperature $T$ taken from [14].

\footnotetext{
${ }^{3}$ In this instance, "wring" refers to the process whereby gauge blocks are twisted as their optically smooth surfaces are in contact so as to remove the intervening air layers.
} 
It is apparent that the two sets of data agree within the experimental uncertainty. (The error bar is smaller on the $25^{\circ} \mathrm{C}$ data point than on the higher temperatures due to the fact that more repeat runs were performed, which reduced the uncertainty for that data point.) This demonstrates several key conclusions regarding the capacitance cell. First, the limitations of the previous design have been eliminated; silicon and conducting samples can be measured. Second, the results show that the capacitance cell produces data that agree with literature data. Finally, we have further demonstrated the advantage of our technique for measurement of thin samples over commercially available TMAs. The validity of this statement can be shown by considering the results of a round robin study. This study was performed among researchers at NIST, IBM Endicott, DEC, Microelectronics and Computer Technology Corporation, Naval Surface Warfare Center-Crane Division, CALCE Electronic Products and Systems Center at the University of Maryland, Cornell University, University of Texas at Austin, Purdue University, and the Semiconductor Research Corporation (SRC) on the measurement of the CTE of single crystal $\langle 100\rangle$ silicon using various commercial TMAs [15]. A 1.1765-mm thick sample of $\langle 100\rangle$ single crystal silicon was used by all participants. All reported values for the CTE of silicon were below the literature values for the corresponding temperature ranges by $15 \%$ to $40 \%$. Our sample was approximately half as thick as their sample, yet our values are within the experimental error. (It should be recalled that our total precision is independent of actual thickness and the main error is due to electrode/sample interfacial effects. Therefore, had we used the thicker sample, as was used in the round robin study, the error in our results would have been reduced.)

In closing, it should be mentioned that since silicon was the "worst case" scenario for the new capacitance cell, it was deemed unnecessary to perform measurements on single crystals of a metallic sample which have a much higher CTE. However, a single measurement was taken on the silicon by connecting the braids from the high and low terminals together shorting the two guard rings as if it were done by a metallic sample. The measured capacitance was unchanged; this therefore demonstrated that conducting materials can be measured.

\section{CONCLUSIONS}

We have presented the designs and implementation of our capacitance cell for the measurement of conducting and semiconducting materials (as well as dielectrics). The thermal expansion data, obtained with the new version of our capacitance cell, on $\langle 100\rangle$ p-type doped single crystal silicon have demonstrated both the ability of the cell to measure silicon and conducting samples and the ability of the cell to provide accurate CTE data on these types of materials. As a result, it is apparent that this metrology can also be applied to thin polymer films deposited on silicon substrates. Furthermore, this cell can also be used to study the hygrothermal expansion (swelling due to the presence of moisture) by utilizing the data reduction techniques described in III. Accordingly, this technique should be especially useful to the microelectronics packaging industry for the characterization of inner layer dielectrics as well as composite structures.

\section{ACKNOWLEDGMENT}

The authors would like to thank Dr. J. R. Ehrstein in the Semiconductor Electronics Division at NIST for providing the silicon sample.

\section{REFERENCES}

[1] R. A. Storer, Ed., Annual Book of ASTM Standards. West Conshohocken, PA: American Society for Testing of Materials, 1997, vol. 14.02 , p. 548.

[2] IPC-TM-650 Test Methods Manual, The Institute for Interconnecting and Packaging Electronic Circuits, Northbrook, IL.

[3] W. Wu, J. H. van Zanten, and W. J. Orts, "Film thickness dependent thermal-expansion in ultrathin poly(methyl methacrylate) films on silicon," Macromolecules, vol. 28, pp. 771-774, 1995.

[4] J. L. Keddie, R. A. L. Jones, and R. A. Cory, "Size-dependent depression of the glass-transition temperature in polymer films," Europhys. Lett., vol. 27, pp. 59-64, 1994.

[5] P. S. Ho, T. W. Poon, and J. Leu, "Molecular-structure and thermal-mechanical properties of polymer thin-films," J. Phys. Chem. Solids, vol. 55, pp. 1115-1124, 1994.

[6] G. D. Sao and H. V. Tiwary, "Thermal expansion of poly(vinylidene fluoride) films," J. Appl. Phys., vol. 53, pp. 3040-3043, 1982.

[7] H. N. Subrahmanyam and S. V. Subramanyam, "Thermal expansion of irradiated polystyrene," J. Mater. Sci., vol. 22, pp. 2079-2082, 1987.

[8] H. M. Tong, H. K. D. Hsuen, K. L. Saenger, and G. W. Su, "Thickness direction coefficient of thermal expansion measurement of thin polymer films," Rev. Sci. Instrum., vol. 62, pp. 422-430, 1991.

[9] M. Schen, F. I. Mopsik, W. Wu, W. E. Wallace, N. C. Beck Tan, G. T. Davis, and W. Guthrie, "Advances in the measurement of polymer CTE: Micrometer-to atomic-scale measurements," Polym. Prep. (Amer. Chem. Soc. Div. Polym. Chem.), vol. 37, pp. 180-182, 1996.

[10] C. R. Snyder and F. I. Mopsik, "A precision capacitance cell for measurement of thin film out-of-plane expansion. I. Thermal expansion," Rev. Sci. Instrum., vol. 69, pp. 3889-3895, 1998.

[11] — "A precision capacitance cell for measurement of thin film out-ofplane expansion. II. Hygrothermal expansion," Rev. Sci. Instrum., vol. 70, pp. 2424-2431, 1999.

[12] E. R. Cohen and B. N. Taylor, "The fundamental physical constants," Phys. Today, vol. 50, pp. BG7-BG11, 1997.

[13] A. A. Maryott and F. Buckley, "Table of dielectric constants and electric dipole moments of substances in the gaseous state,", NBS Circular 537, 1953.

[14] C. A. Swenson, "Recommended values for the thermal expansivity of silicon from 0 to 1000 K," J. Phys. Chem. Ref. Data, vol. 12, pp. $179-182,1983$.

[15] M. Schen et al., "An industry/government/university partnership: Measuring sub-micrometer strain in polymer films," in Proc. Tech. Conf., IPC Printed Circuits Expo., Apr. 24-27, 1994.

Chad R. Snyder (M'99) was born in York, PA, in 1969. He received the B.S. degree in chemistry from Shippensburg University, Shippensburg, PA, in 1991, and the Ph.D. degree in physical chemistry from Virginia Polytechnic Institute and State University, Blacksburg, in 1995.

In 1996, he accepted a National Research Council Postdoctoral Research Fellowship at the National Institute of Standards and Technology (NIST), Gaithersburg, MD, in the Electronics Applications Group of the Polymers Division in the Materials Science and Engineering Laboratory. He is currently a full-time staff member in the Electronics Materials Group with interests in the properties of thin polymer films, electromagnetic properties of nanoparticle dispersions, time-domain dielectric spectroscopy, dielectric relaxation of polymers, and polymer crystallization.

Frederick I. Mopsik received the Ph.D. degree in physical chemistry from Brown University, Providence, RI, 1964.

He recently retired from the National Institute of Standards and Technology (NIST), Gaithersburg, MD, after 35 years of service. His active areas of research have included the development of precision measurements on dielectric materials over a frequency range of $10^{-4}$ to greater than $10^{9} \mathrm{~Hz}$ and over broad ranges of temperature and pressure. Some of these include precision time-domain measurements at low frequencies, the measurement of low loss materials down to $4 \mathrm{~K}$, and the measurement of simultaneous PVT and dielectric measurements at pressures up to $200 \mathrm{mPa} \cdot \mathrm{s}$. 